\title{
Miocardiopatía hipertrófica: ¿cómo evaluamos en 2018 el riesgo de muerte súbita?
}

\author{
Dres. Alejandro Estrada1,2,3,4, Eduardo Brenna, ${ }^{2,4}$, Alejandro Contreras ${ }^{2,4}$, \\ Marcos Amuchástegui ${ }^{2,3,4}$, Carlos Estrada², Andrés Caeiro ${ }^{1,2,3,4}$, Oscar Salomone ${ }^{2,4}$
}

\begin{abstract}
Resumen
La miocardiopatía hipertrófica (MCH) es una enfermedad frecuente del miocardio, asociada a muerte súbita cardíaca (MSC). Las principales causas de muerte en MCH son la MSC, la insuficiencia cardíaca y el tromboembolismo. La fibrilación ventricular (FV) es la causa más común de MSC, seguida de asistolia, actividad eléctrica sin pulso y bloqueo auriculoventricular completo. El sustrato de estas arritmias complejas es la hipertrofia asociada a desorganización miofibrilar y fibrosis, que facilitan el desarrollo de arritmias reentrantes y secundarias a actividad desencadenada. Dado que la FV puede ser tratada con el implante de un desfibrilador automático implantable (DAI), la identificación de los pacientes que presentan mayor riesgo se transforma en la principal preocupación del cardiólogo tratante. La indicación de implante de DAI es clara en la prevención secundaria de MSC, pero es compleja en el resto de los pacientes que no han presentado FV o taquicardia ventricular sostenida, lo que configura la prevención primaria de MSC. Debemos estratificar la MCH con el uso de los factores de riesgo derivados de datos clínicos y estudios complementarios. Los principales factores de riesgo relacionados a MSC son: edad temprana al diagnóstico, antecedentes heredofamiliares de MSC, síncope inexplicado, taquicardia ventricular no sostenida, hipotensión arterial inducida durante la prueba de esfuerzo, obstrucción del tracto de salida del ventrículo izquierdo, hipertrofia ventricular izquierda severa, agrandamiento auricular izquierdo y captación de realce tardío en la resonancia magnética. El uso adecuado de los dos principales modelos de estratificación de riesgo actualmente disponibles nos ayuda a estimar el riesgo individual en cada paciente y proceder en consecuencia.
\end{abstract}

Palabras clave:

MIOCARDIOPATÍA HIPERTRÓFICA

MUERTE SÚBITA CARDÍACA

ARRITMIAS CARDÍACAS

\section{Hypertrophic cardiomyopathy: how do we evaluate the risk of sudden death in 2018?}

\section{Summary}

Hypertrophic cardiomyopathy is one of the most frequent cardiac muscle disorders associated with sudden cardiac death, heart failure and thromboembolism. Ventricular fibrillation is the most common cause of sudden cardiac death in hypertrophic cardiomyopathy, followed by asystole, pulseless electrical activity, and complete atrioventricular block. The substrate behind these complex arrhythmias is the hypertrophy due to myocardial disarray and fibrosis, facilitating the development of reentrant arrhythmias and triggered activity. An implanted cardioverter defibrillator is the treatment of choice, so the identification of high risk patients for ventricular fibrillation is crucial. Implanted cardioverter defibrillator indication is well established in sudden cardiac death secondary prevention, but not in patients without evidence of ventricular fibrillation or sustained ventricular tachycardia, which configures the sudden cardiac death primary prevention group. Identification of sudden cardiac death risk factors is derived from clinical data, complementary studies and validated in risk stratification models. These are: early age at diagnosis, family history of sudden death, unexplained syncope, non-sustained ventricular tachycardia, abnormal blood pressure response with exercise, left ventricular outflow tract obstruction, severe left ventricular hypertrophy, left atrial enlargement and late gadolinium enhancement on cardiac magnetic resonance imaging. The proper use of two available main risk stratification models helps us to estimate the individual patient's risk and to proceed consequently.

Key words:

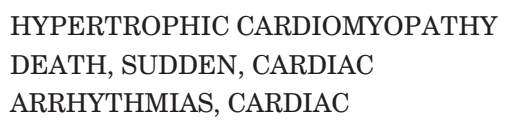

1. Unidad de Arritmias y Electrofisiología Cardíaca. Servicio de Cardiología. Hospital Privado Universitario de Córdoba, Argentina.

2. Servicio de Cardiología. Hospital Privado Universitario de Córdoba, Argentina.

3. Servicio de Hemodinamia. Hospital Privado Universitario de Córdoba, Argentina.

4. Instituto Universitario de Ciencias Biomédicas de Córdoba, Argentina.

Correspondencia: Alejandro Estrada. Correo electrónico: aleestrada77@hotmail.com

Los autores declaran no tener conflictos de intereses. 


\section{Introducción}

La miocardiopatía hipertrófica ( $\mathrm{MCH})$ es una enfermedad frecuente del miocardio asociada a muerte súbita cardíaca (MSC) (1). Su prevalencia en la población general es de un caso cada 500 personas, pero podría ser mayor si el diagnóstico lo realizamos con pruebas genéticas (un caso cada 200 personas). Esta enfermedad se define por el hallazgo de hipertrofia ventricular izquierda (HVI) no explicada por condiciones anormales de sobrecarga, y se asocia frecuentemente a obstrucción del tracto de salida del ventrículo izquierdo (OTSVI) debido al movimiento anterior sistólico de la válvula mitral ${ }^{(1-3)}$. Desde el punto de vista histológico se caracteriza por hipertrofia cardiomiocitaria, desorganización miofibrilar ( $d i$ sarray), fibrosis miocárdica y enfermedad de la microvasculatura $^{(3-4)}$. El origen genético lo encontramos principalmente por mutaciones en genes sarcoméricos (40\%-60\% de los casos), aunque existen otros factores que pueden modular el fenotipo de tal manera que muchos de los portadores de mutaciones patogénicas no exhiben la enfermedad ${ }^{(5)}$.

Las principales causas de muerte en la $\mathrm{MCH}$ son la MSC, la insuficiencia cardíaca y el tromboembolismo ${ }^{(3,5)}$. La estimación de la mortalidad cardiovascular real de este grupo de pacientes ha cambiado en los últimos años, ya que en estudios iniciales de grandes centros de referencia era del 6\% anual. En contraste, estudios recientes demuestran un curso clínico más favorable, con una mortalidad cardiovascular en torno al $2 \%$ anual, con MSC del $0,8 \%$ anual predominando en la adultez temprana ${ }^{(3,6-8)}$. El desfibrilador automático implantable (DAI) ha demostrado prevenir la MSC, por lo cual la identificación de los pacientes que presentan mayor riesgo se transforma en la principal preocupación al momento de enfrentarse con esta patología ${ }^{(3)}$.

\section{Causas de muerte súbita cardíaca}

Las causas de MSC descriptas son fibrilación ventricular (FV) espontánea, asistolia, actividad eléctrica sin pulso y bloqueo auriculoventricular completo $^{(5,9-11)}$. Se obtuvo información valiosa de los dispositivos cardíacos (registros de electrogramas en marcapasos y DAI) y de los infrecuentes registros electrocardiográficos, identificando a la FV como la causa más frecuente de MSC en MCH. El sustrato de estas arritmias complejas es la hipertrofia asociada a desorganización miofibrilar que facilita el desarrollo de arritmias reentrantes y secundarias a actividad desencadenada (posdespolarizaciones) $)^{(5,12)}$. A pesar de este sustrato arritmogénico, la real incidencia de MSC es baja, debido a que las arritmias ventriculares son inducidas cuando se presentan condiciones vulnerables como la OTSVI y la isquemia miocárdi$\mathrm{ca}^{(3)}$ (figura 1). Por otra parte, la baja incidencia de MSC debe ser matizada por la catástrofe social que significa la temprana edad en la que ocurren estos eventos fatales, por lo cual es crucial desarrollar estrategias de estratificación de riesgo que permitan identificar precozmente y con certeza aquellos pacientes más vulnerables.

\section{Estratificación de riesgo}

Lo primero que debemos hacer en la evaluación inicial de la MCH es identificar aquellos pacientes con alto riesgo de MSC que se beneficiarán del tratamiento profiláctico con DAI. La selección es sencilla cuando los pacientes han presentado episodios de arritmias ventriculares sostenidas, configurando el grupo de mayor riesgo de recurrencias arrítmicas (prevención secundaria de MSC). El tamizaje no es sencillo en el resto de los pacientes y nos obliga a acudir a diferentes variables clínicas para lograr la mejor selección de los pacientes en riesgo, lo que llamamos prevención primaria de MSC. Toda esta información fue derivada de estudios observacionales, retrospectivos y de cohorte que usaron análisis multivariados de sobrevivencia para examinar la asociación de diferentes características clínicas con la MSC ${ }^{(3,5,8)}$ (figura 2).

Diferentes estudios investigaron el riesgo de MSC asociado a ciertas combinaciones de factores de riesgo mayores, encontrando un progresivo empeoramiento del pronóstico con la sumatoria de estas variables clínicas ${ }^{(13)}$.

La evidencia actual sugiere estudiar a adolescentes y adultos con una estratificación de riesgo que incluya el relevamiento de antecedentes heredofamiliares de MSC o MCH y la realización de electrocardiograma, Holter de 48 horas, prueba de esfuerzo y ecocardiograma o resonancia magnética $(\mathrm{RM})^{(5,14-15)}$. Mediante este abordaje intentaremos detectar los diferentes factores de riesgo que describiremos a continuación.

\section{Factores de riesgo de muerte súbita}

\subsection{Edad}

Se evalúa la edad al momento del diagnóstico de MCH. Estudios iniciales han demostrado que los adolescentes y adultos jóvenes presentan riesgo aumentado de $\mathrm{MSC}^{(5,16)}$. Otras investigaciones recientes de sobrevivencia muestran una reducción de riesgo con el incremento de la edad de los pacientes afectados. Sin embargo, la edad no sería un factor de riesgo mayor, siendo superado por otros como la 


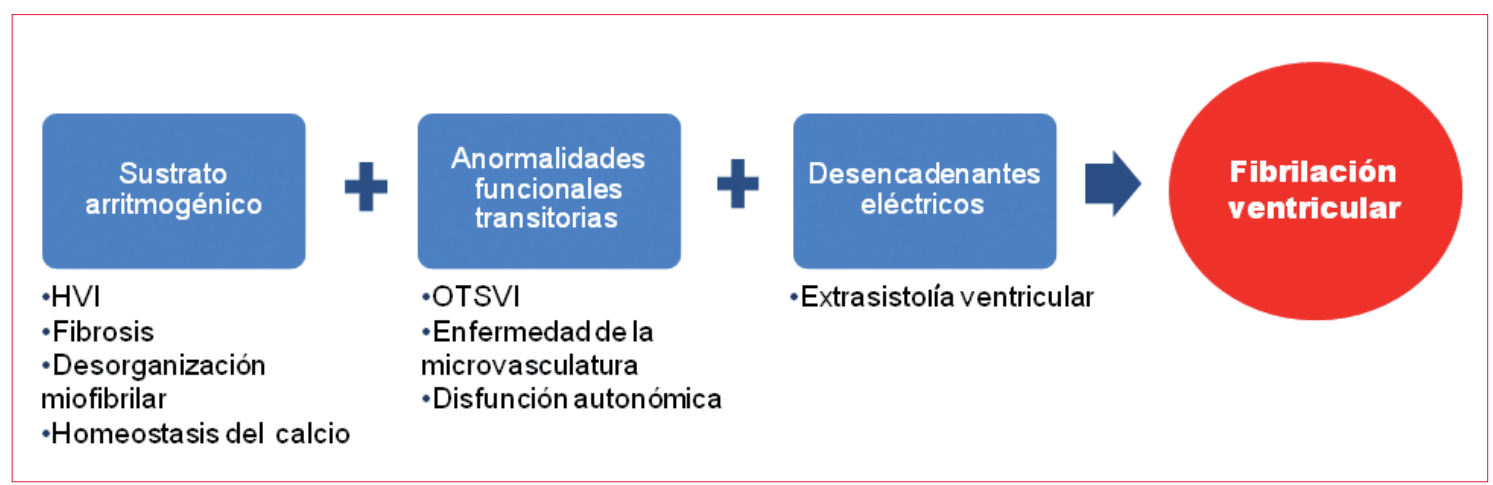

Figura 1. Condiciones para la inducción de fibrilación ventricular en miocardiopatía hipertrófica. HVI: hipertrofia ventricular izquierda; OTSVI: obstrucción del tracto de salida del ventrículo izquierdo

taquicardia ventricular no sostenida (TVNS), la HVI severa y el síncope inexplicado ${ }^{(1,17)}$.

\subsection{Antecedentes heredofamiliares de muerte súbita}

Se considera un factor de riesgo cuando la MSC se presenta en un familiar de primer grado antes de los 40 años sin diagnóstico de $\mathrm{MCH}$ o a cualquier edad con diagnóstico de $\mathrm{MCH}^{(5)}$. Es un elemento débil en la estratificación de riesgo, demostrando un aumento del 27\% del riesgo relativo de MSC en un metaanálisis, con la salvedad de que se utilizaron diferentes definiciones de antecedentes heredofamiliares de $\mathrm{MSC}^{(13)}$. Otro dato que complejiza la interpretación es que el diagnóstico de MSC no es claramente definido por los testigos del evento o en los certificados de defunción ni se realizan frecuentes exámenes post mórtem ${ }^{(3)}$.

\subsection{Síncope}

El síncope es frecuente en pacientes con $\mathrm{MCH}$, pero su interpretación no es sencilla debido a su multi-

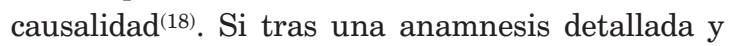
realización de estudios complementarios no llegamos a explicar el mismo, se considera un factor de alto riesgo de MSC. Si los episodios ocurren en un intervalo menor de seis meses se asocian a un mayor riesgo ${ }^{(5,17)}$. Los estudios demuestran diferentes resultados, pero en promedio la presencia de síncope inexplicado se asocia a un aumento significativo de MSC (HR 2,68; IC95\%: 0,97-4,38)(13).

\subsection{Taquicardia ventricular no sostenida}

Definimos la TVNS como la presencia de más de tres latidos consecutivos a más de 120 latidos por minuto durante menos de 30 segundos, en episodios repetidos (múltiples), registrados en un estudio de Holter de 24 o 48 horas. La presencia de TVNS se asocia a MSC, siendo uno de los factores de riesgo mayores en la estratificación de riesgo en $\mathrm{MCH}$.
Tanto es así que los pacientes menores de 30 años con MCH y TVNS en Holter tienen un riesgo cuatro veces mayor de eventos graves ${ }^{(3,13,18)}$. Por otra parte, las arritmias ventriculares inducidas por el ejercicio, presentes en el $2 \%$ de los casos, se asocian de forma independiente a $\operatorname{MSC}^{(19)}$.

\subsection{Respuesta anormal de la presión arterial durante el ejercicio}

Durante la prueba de esfuerzo (ergometría en cinta sinfín) un tercio de los pacientes con $\mathrm{MCH}$ presenta una respuesta anormal de la presión arterial que se caracteriza por progresiva hipotensión o incapacidad para aumentar la presión arterial sistólica (más de $20 \mathrm{mmHg}$ desde el reposo al máximo esfuerzo o reducción de $20 \mathrm{mmHg}$ durante el ejercicio). Este fenómeno se debe a una caída inapropiada de la resistencia vascular sistémica sumada a una reducción del gasto cardíaco ${ }^{(5,20,21)}$. Se asocia a MSC en menores de 40 años, pero no es un factor de riesgo independiente en los análisis multivariados ${ }^{(5,22)}$. Una revisión sistemática reciente demuestra un aumento del riesgo relativo de MSC en un $30 \%(13)$.

\subsection{Obstrucción del tracto de salida del ventrículo izquierdo}

La OTSVI es producida por el movimiento anterior sistólico de la válvula mitral hacia el tracto de salida, lo cual crea una barrera física que impide el flujo desde el ventrículo hacia la aorta durante la sístole. El mecanismo por el cual aumenta la MSC es mediante la severa reducción del gasto cardíaco, llevando a la disociación electromecánica o induciendo arritmias ventriculares complejas debido a isquemia miocárdica secundaria, consecuencia de una elevada presión de fin de diástole del ventrículo izquierdo $^{(3)}$.

La OTSVI se define como la presencia de gradiente pico mayor a $50 \mathrm{mmHg}$ medido por ecocardiograma Doppler, tanto de forma basal en reposo 


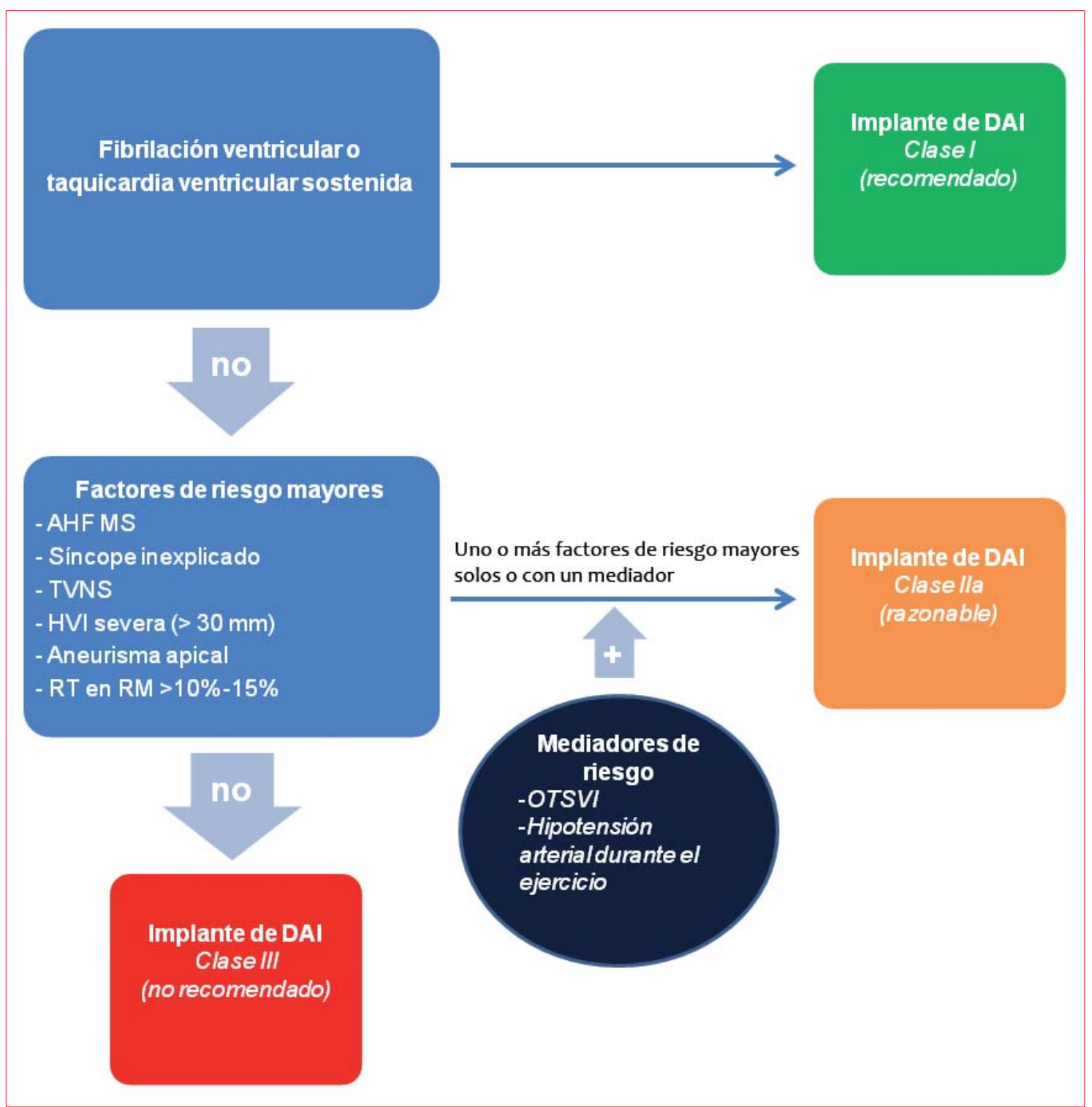

Figura 2. Indicaciones de implante de DAI como prevención primaria y secundaria de muerte súbita según recomendaciones de las guías norteamericanas 2011(modificado). DAI: desfibrilador automático implantable; AHF MS: antecedentes heredofamiliares de muerte súbita; TVNS: taquicardia ventricular no sostenida; HVI: hipertrofia ventricular izquierda; RT en RM: realce tardío de gadolinio en resonancia magnética cardíaca; OTSVI: obstrucción del tracto de salida del ventrículo izquierdo.

como provocado con la maniobra de Valsalva. Un gradiente mayor a $30 \mathrm{mmHg}$ indica OTSVI, pero cuando es mayor a $50 \mathrm{mmHg}$ constituye un dato clínicamente significativo.

Los resultados de los estudios demuestran una asociación entre OTSVI y MSC. Sin embargo, quedan algunas preguntas sin resolver, como el impacto de la reducción septal (quirúrgica o con ablación alcohólica) sobre la disminución del riesgo de $\operatorname{MSC}^{(23,24)}$.

\subsection{Hipertrofia ventricular izquierda severa}

La HVI severa se asocia a MSC debido a su efecto deletéreo en la estructura miocárdica, sumado a la demanda aumentada de oxígeno, resistencia vascular coronaria y a la densidad capilar ${ }^{(1,25)}$. Este sustrato suele asociarse a otros factores de riesgo, como la TVNS y las arritmias ventriculares inducidas por el ejercicio $^{(1)}$.

Se define la HVI severa como la medición ecocardiográfica del grosor de pared ventricular mayor a $30 \mathrm{~mm}$. Esta definición nos hace ver el problema desde una interpretación binaria de los resultados (mayor o menor de $30 \mathrm{~mm}$ ) y nunca como un fenómeno continuo, como muchos autores consideran que debería valorarse. Es decir que nos preguntaríamos si un paciente que tiene $29 \mathrm{~mm}$ de grosor de pared tiene menor riesgo, en comparación con otro paciente que tiene $30 \mathrm{~mm}$, máxime conociendo la 


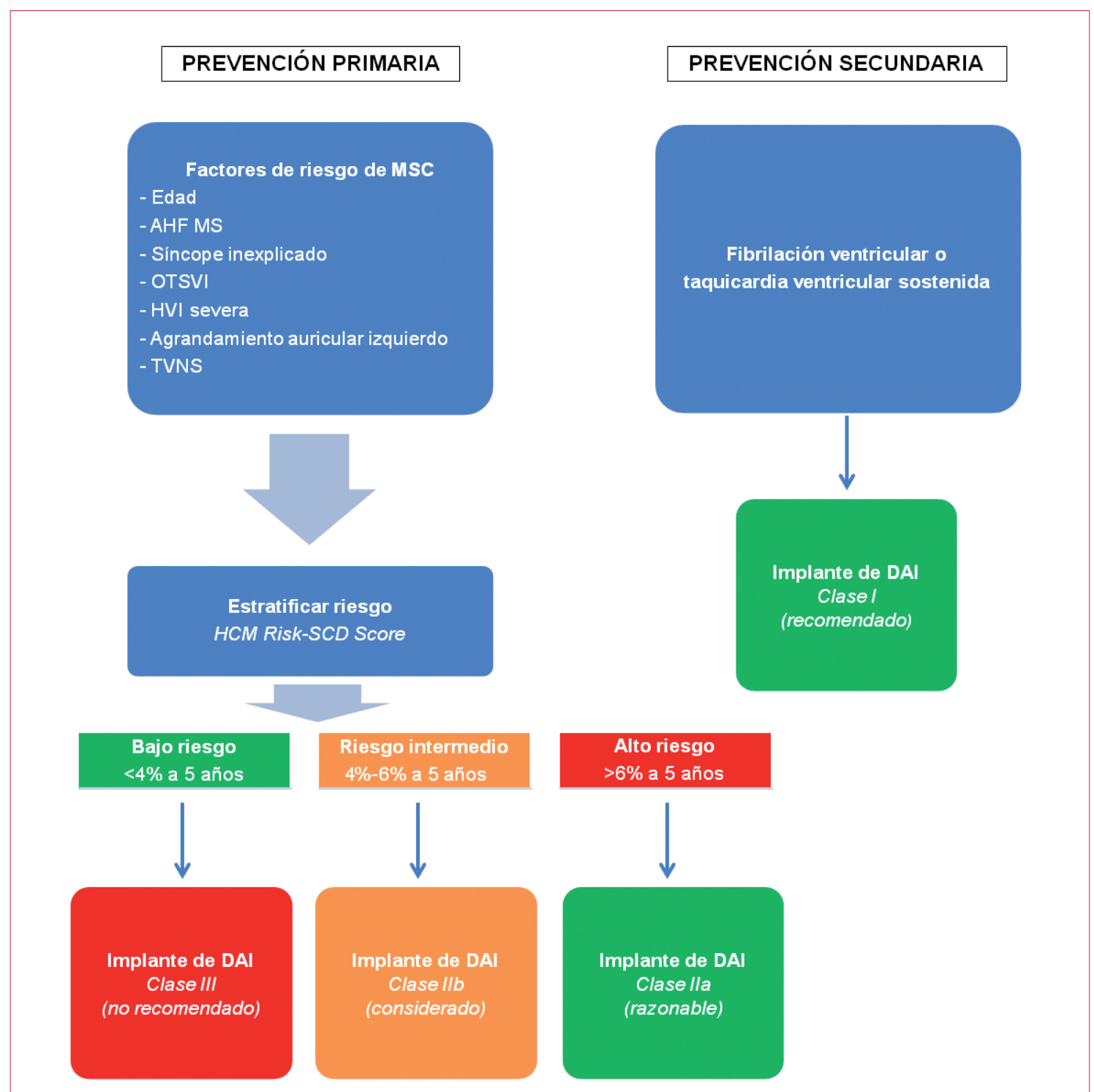

Figura 3. Indicaciones de implante de DAI como prevención primaria y secundaria de muerte súbita según recomendaciones de las guías europeas 2014 (Sociedad Europea de Cardiología, modificado). AHF MS: antecedentes heredofamiliares de muerte súbita; OTSVI: obstrucción del tracto de salida del ventrículo izquierdo; HVI: hipertrofia ventricular izquierda; TVNS: taquicardia ventricular no sostenida; DAI: desfibrilador automático implantable.

variabilidad interobservador de las mediciones ecocardiográficas. Estas reflexiones no hacen más que revelar el valor relativo y las potenciales limitaciones que se derivan de nuestra estratificación de riesgo ${ }^{(5,6,25,26)}$.

En un metaanálisis se relacionó la HVI mayor a $25 \mathrm{~mm}$ con muerte de causa cardíaca o MSC, triplicando el riesgo relativo de presentar estos eventos $(3.10[\mathrm{IC} 95 \%: 1,81-4,40])^{(13)}$.

\subsection{Diámetro de aurícula izquierda}

El diámetro de la aurícula izquierda medido por ecocardiografía en modo M, en un eje largo paraesternal izquierdo, es un factor de riesgo conocido. Sin embargo, no se valoró el valor pronóstico del área ni del volumen de la aurícula izquierda, lo cual plantea limitaciones en la evaluación de este factor de riesgo. Se observó una asociación entre el diámetro aumentado de la aurícula izquierda y MSC, al igual que con la presencia de fibrilación auricular ${ }^{(3,5)}$.

\subsection{Realce tardío de gadolinio en la resonancia magnética cardíaca}

El realce tardío (RT) de gadolinio identificado en la $\mathrm{RM}$ tiene valor predictivo como un factor de riesgo de MSC en niños y adultos con $\mathrm{MCH}^{(27)}$. Este hallazgo se asocia a HVI severa, disfunción sistólica ventricular izquierda y TVNS ${ }^{(1,28)}$. Algunos autores sugieren 
que el RT es el predictor más potente de MSC en relación con otros factores de riesgo clásicos, aunque con resultados controvertidos, ya que la definición de presencia o ausencia de RT es un resultado binario, $\mathrm{y}$ es conocido que a mayor cantidad de RT, mayor es la tasa de eventos ${ }^{(29,30)}$. Intentando establecer cuál es el porcentaje de masa ventricular afectada por el RT se sugieren valores de corte de $10 \%$ a $15 \%$, que evidencian una severa fibrosis miocárdica ${ }^{(27,31)}$. Por otra parte, debemos destacar que la fibrosis miocárdica es un fenómeno dinámico, demostrándose un aumento del RT al repetir la RM a los dos años; siendo más acentuado este aumento en la hipertrofia apical y en aquellos pacientes que han deteriorado su clase funcional $^{(32)}$. El agregado del RT a los factores de riesgo clásicos permitiría reestratificar aquellos pacientes de riesgo bajo o intermedio en los que no deberíamos implantar un DAI. Sin embargo, aún no se dispone de evidencias sólidas que permitan validar esta hipótesis. Por el contrario, los pacientes con $\mathrm{MCH}$ sin factores de riesgo clásicos y sin $\mathrm{RT}$ tienen un curso clínico benigno, con una mortalidad similar a la de los pacientes sin $\mathrm{MCH}^{(27,29)}$.

\section{Modelos para estimar muerte súbita cardíaca}

La estimación de MSC en la MCH presenta limitaciones ya que se basa en el riesgo aceptable, asumiendo que los pacientes de bajo riesgo tienen menos eventos graves, por lo cual no deberían recibir un DAI. Sin embargo, en este grupo de bajo riesgo hay pacientes que escapan de nuestras predicciones y debutan con MSC sin la posibilidad de recibir el DAI profiláctico a tiempo. Las recomendaciones de implante de DAI están basadas en estudios observacionales, retrospectivos y de cohorte que miden la asociación de ciertas características clínicas con el pronóstico(5) $^{(5)}$ Esta situación nos plantea una clara diferencia con las recomendaciones para prevención primaria de MSC en las miocardiopatías isquémica y dilatada, donde la indicación de DAI está basada en estudios clínicos randomizados prospectivos que le confieren una evidencia más sólida ${ }^{(33-35)}$.

En la actualidad contamos con dos abordajes principales para estratificar riesgo de MSC en $\mathrm{MCH}$, sugeridos por las guías de práctica clínica norteamericanas y europeas ${ }^{(5,14)}$. Las guías norteamericanas sugieren implantar DAI a los pacientes con $\mathrm{MCH}$ que presenten al menos un factor de riesgo mayor de MSC (síncope inexplicado, HVI severa, antecedentes heredofamiliares de MSC, múltiples episodios de TVNS, RT mayor a $15 \%$ en la RM cardíaca y aneurisma apical)(14,31). Esta estrategia es efectiva y sensible para identificar a la mayoría de los pacientes con riesgo aumentado de arritmias ventriculares complejas (figura 2).

Por otra parte, la estratificación de riesgo sugerida por las guías europeas incluye la utilización de un modelo de predicción de riesgo de MSC validado, que utiliza los factores de riesgo habituales, como la edad al momento del diagnóstico, antecedentes heredofamiliares de muerte súbita, síncope inexplicado, OTSVI, HVI severa y diámetro de la aurícula izquierda ${ }^{(3,36)}$ (figura 3). Este modelo estima un riesgo de MSC a cinco años estratificando en tres grupos de riesgo: bajo ( $\leq 4 \%$ anual), intermedio (4\%-6\%) y alto $(\geq 6 \%)$. Se recomienda implante de DAI como prevención primaria de MSC en los pacientes clasificados como de alto riesgo. Los resultados de este modelo se obtienen fácilmente mediante el llenado sencillo y rápido de datos clínicos en una página en internet (HCM Risk-SCD model). El modelo no debe utilizarse en menores de 16 años, atletas de elite y portadores de enfermedades infiltrativas o metabólicas (enfermedad de Anderson-Fabry o síndrome de Noonan, por ejemplo) $)^{(3,5,36,37)}$.

Es necesario aclarar que, al tratarse de una enfermedad con cambios dinámicos y progresivos, es fundamental realizar un seguimiento adecuado con reestratificación del riesgo de MSC en forma anual si el paciente permanece asintomático o cuando cambie su situación clínica (por ejemplo, tras un síncope). Los pacientes de riesgo bajo o intermedio deben ser instruidos con pautas de alarma y en la necesidad de controles médicos anuales (consulta y estudios complementarios) ${ }^{(5)}$.

Finalmente, merece la pena reflexionar sobre los valores establecidos para estratificación de riesgo de MSC sobre la base del "riesgo aceptable" que surge de opiniones de expertos. Si el implante de DAI no tuviera complicaciones, le implantaríamos este dispositivo a todos los pacientes con $\mathrm{MCH}$ sin importar si su riesgo es bajo, intermedio o alto. $\mathrm{Al}$ tener potenciales complicaciones debemos seleccionar los pacientes que están en mayor riesgo. En el futuro deberemos rediscutir este valor de "riesgo aceptable", ya que el implante de DAI subcutáneo se plantea como una alternativa con potenciales menores complicaciones a un DAI con electrodos endocavitarios. Quizás el DAI subcutáneo nos permita ser más agresivos e incluir pacientes de menor riesgo.

\section{Prevención de muerte súbita}

\subsection{Restricción de ejercicio}

Los pacientes con MCH deben ser aconsejados para no realizar deporte competitivo ni actividad física 
intensa, ya que las arritmias ventriculares malignas pueden desencadenarse durante estas prácticas. Tanto es así que la MCH es la causa cardiovascular más frecuente de MSC en atletas jóvenes, representando un tercio de estos eventos ${ }^{(38,39)}$. Las recomendaciones para elegibilidad y descalificación de atletas competitivos establecen que aquellos pacientes con expresión clínica y diagnóstico inequívoco de $\mathrm{MCH}$ no deben realizar deportes competitivos, independientemente de si tienen o no factores de riesgo o han sido intervenidos (ablación septal con alcohol o septostomía quirúrgica) o tratados con drogas antiarrítmicas ${ }^{(40)}$.

A pesar de estas recomendaciones, es infrecuente registrar taquicardias ventriculares sostenidas durante el ejercicio sumado a que la mayoría de las terapias de DAI ocurren en ausencia de actividad física intensa, lo cual pone en duda la restricción del ejercicio intenso por conflictos en la interpretación de esta información y por el impacto potencialmente mayor en la calidad de vida(5,19,41). El mecanismo exacto por el cual el ejercicio físico intenso induce MSC no está claro, pero se postula que podría jugar un rol importante la hipotensión arterial, el aumento de la OTSVD y la isquemia miocárdica inducidas por el ejercicio ${ }^{(3)}$.

\subsection{Drogas antiarrítmicas}

El uso de drogas antiarrítmicas fue estudiado para la prevención de MSC en MCH sin resultados positivos. Por otra parte, hasta el momento no se han realizado estudios randomizados con antiarrítmicos y solo tenemos resultados de pequeños estudios observacionales que han demostrado una discreta reducción de la TVNS en Holter y aumento del umbral de $\mathrm{FV}^{(42,43)}$. En definitiva, no hay evidencia para utilizar antiarrítmicos con el fin de reducir la MSC en la $\mathrm{MCH}^{(3,5)}$.

\subsection{Desfibrilador automático implantable}

El tratamiento actual para evitar la MSC en $\mathrm{MCH}$ es el implante de DAI (patrón oro), tanto en prevención primaria como secundaria. Esta indicación surge de la información publicada que demuestra que la MSC es rara en portadores de DAI y que estos pacientes reciben terapias apropiadas para terminar arritmias ventriculares potencialmente morta$\operatorname{les}^{(1,5,44,45)}$. A pesar de estos claros beneficios, el implante de DAI se asocia a una considerable morbilidad derivada de las complicaciones relacionadas al implante $(2 \%-25 \%)$ y de las terapias inapropiadas (15\%-32\%), máxime por el hecho de que muchos de los pacientes en riesgo son niños, adolescentes o jóvenes adultos que deberán portar este dispositivo durante toda su vida ${ }^{(1,5,46)}$. Por otra parte, hay que destacar que los pacientes que reciben un DAI presentan frecuentemente una baja calidad de vida, problemas psicológicos y restricciones laborales ${ }^{(1,47)}$. Finalmente, se agrega una cuestión no menos importante, que es el alto costo que presenta el DAI para el sistema de salud y los requerimientos de seguimiento médico a largo plazo.

\section{Zona de incertidumbre}

Quedan muchos problemas sin resolver que plantean dudas sobre el tratamiento profiláctico con DAI, entre los que destacamos el riesgo residual de MSC en pacientes postseptostomía quirúrgica o con alcohol. Al tratarse de una enfermedad con múltiples alteraciones cardíacas (entre las más importantes fibrosis y desorganización miofibrilar), la simple reducción del volumen del septum interventricular no alcanzaría para cambiar de forma significativa el riesgo de arritmias ventriculares complejas.

Otro tema sin resolver, pero en franco avance, es el valor de las pruebas genéticas en los nuevos algoritmos de estratificación de riesgo. A pesar de los grandes progresos en el estudio de la patogenia de la $\mathrm{MCH}$, los conocimientos actuales son insuficientes para predecir los fenotipos que expresará cada paciente. En la expresión fenotípica influyen los genes causales, los genes modificadores y los factores ambientales, por lo cual el simple conocimiento de una mutación no otorga información válida y confiable sobre el riesgo del paciente. Quizás en el futuro podamos adoptar medidas preventivas en los pacientes portadores de mutaciones genéticas y en sus familias, pero no por el momento ${ }^{(48)}$.

\section{Conclusiones}

La MCH es una enfermedad frecuente del miocardio asociada a MSC. Las principales causas de muerte en $\mathrm{MCH}$ son la MSC, la insuficiencia cardíaca y el tromboembolismo. La FV es la causa más común de MSC y puede ser prevenida con el implante de DAI, por lo cual la identificación de los pacientes que presentan mayor riesgo constituye la principal preocupación. La indicación del implante de DAI es sencilla e indiscutible en los pacientes que han sobrevivido a una FV o a una taquicardia ventricular sostenida (prevención secundaria de MSC). Esta indicación es compleja en el resto de los pacientes que no han presentado un evento grave (prevención primaria de MSC), teniendo la difícil tarea de estratificar el riesgo de MSC con el uso de los factores de riesgo derivados de los datos clínicos y de los estudios complementarios. El uso adecuado de los dos 
Resumen conceptual

1. La FV es la causa más común de MSC y puede ser prevenida con el implante de un DAI.

2. Todos los pacientes con MCH deberían ser valorados detalladamente con una estratificación de riesgo de MSC.

3. Los pacientes con factores de riesgo de MSC deben recibir un DAI.

4. Los pacientes sin factores de riesgo tienen bajo riesgo de MSC y no requerirían el implante de un DAI, pero deben ser revalorados regularmente para reestratificar su riesgo.

5. Los algoritmos actuales de estratificación de riesgo tienen sus limitaciones, pero son las únicas herramientas de las que disponemos para elegir el mejor tratamiento en cada caso.

6. Sólo una minoría de los pacientes con DAI presentan terapias apropiadas.

7. Los portadores de DAI tienen alto riesgo de sufrir complicaciones relacionadas a este dispositivo (terapias inapropiadas, complicaciones del implante, infecciones, entre otras), por lo cual su indicación debe ser valorada de forma responsable.

principales modelos de estratificación de riesgo actualmente disponibles, nos ayuda a estimar el riesgo individual de cada paciente y actuar en consecuencia.

\section{Bibliografía}

1. O'Mahony C, Elliott P, McKenna W. Sudden cardiac death in hypertrophic cardiomyopathy. Circ Arrhythm Electrophysiol. 2013;6 (2):443-451. doi: 10.1161/CIRCEP.111.962043

2. Elliott P, Andersson B, Arbustini E, Bilinska Z, Cecchi F, Charron P, et al. Classification of the cardiomyopathies: a position statement from the European Society Of Cardiology Working Group on Myocardial and Pericardial Diseases. Eur Heart J.2008;29 (2):270-6.

3. O'Mahony C, Elliott PM. Prevention of sudden cardiac death in hypertrophic cardiomyopathy. Heart 2014;100 (3):254-260. doi: 10.1136/heartjnl2012-301996

4. Varnava AM, Elliott PM, Sharma S, McKenna WJ, Davies MJ. Hypertrophic cardiomyopathy: the interrelation of disarray, fibrosis, and small vessel disease. Heart. 2000;84 (5):476-82.

5. Elliott PM, Anastasakis A, Borger MA, Borggrefe M, Cecchi F, Charron P, et al. 2014 ESC guidelines on diagnosis and management of hypertrophic cardiomyopathy: The task force for the diag- nosis and management of hypertrophic cardiomyopathy of the European Society of Cardiology (ESC). Eur Heart J 2014;35 (39):2733-79. doi: 10.1093/eurheartj/ehu284

6. Elliott PM, Gimeno JR, Thaman R, Shah J, Ward D, Dickie S, et al. Historical trends in reported survival rates in patients with hypertrophic cardiomyopathy. Heart 2006;92 (6):785-91.

7. Maron BJ, Olivotto I, Spirito P, Casey SA, Bellone P, Gohman TE, et al. Epidemiology of hypertrophic cardiomyopathy-related death: revisited in a large non-referral-based patient population. Circulation 2000;102 (8):858-64.

8. Sarrias A, Galve E, Sabaté X, Moya A, Anguera I, Núñez E, et al. Terapia con desfibrilador automático implantable en la miocardiopatía hipertrófica: utilidad en prevención primaria y secundaria. Rev Esp Cardiol. 2015;68 (6):492-6. doi: 10.1016/j.recesp. 2014.05.024

9. Barriales-Villa R, Centurion-Inda R, Fernandez-Fernandez X, Ortiz MF, Perez-Alvarez L, Rodriguez García I, et al. Severe cardiac conduction disturbances and pacemaker implantation in patients with hypertrophic cardiomyopathy. Rev Esp Cardiol. 2010;63 (8):985-8. doi: 10.1016/S0300-8932 (10) $70210-4$

10. Elliott PM, Sharma S, Varnava A, Poloniecki J, Rowland E, McKenna WJ. Survival after cardiac arrest or sustained ventricular tachycardia in patients with hypertrophic cardiomyopathy. J Am Coll Cardiol. 1999;33 (6):1596-601.

11. Joseph S, Balcon R, McDonald L. Syncope in hypertrophic obstructive cardiomyopathy due to asystole. Br Heart J 1972;34 (9):974-6.

12. Romero-Farina R, Candell-Riera J, Aguadé-Bruix S, Castell-Conesa J. Hipertrofia extrema e isquemia subendocárdica silente en la miocardiopatía hipertrófica. Rev Urug Cardiol. 2007; 22 (1): 78-80.

13. Christiaans I, van Engelen K, van Langen IM, Birnie E, Bonsel GJ, Elliott PM, et al. Risk stratification for sudden cardiac death in hypertrophic cardiomyopathy: systematic review of clinical risk markers. Europace 2010;12 (3):313-21. doi: 10.1093/ europace/eup431

14. Gersh BJ, Maron BJ, Bonow RO, Dearani JA, Fifer MA, Link MS, et al. 2011 ACCF/AHA guideline for the diagnosis and treatment of hypertrophic cardiomyopathy: executive summary: a report of the American College of Cardiology Foundation/American Heart Association Task Force on Practice Guidelines. Circulation 2011;124 (24): 2761-96. doi: 10.1161/ CIR.0b013e318223e230

15. Vidal I, Estigarribia J, Báez A, Vidal L. Registro Uruguayo de Miocardiopatía Hipertrófica (RUMHI). Rev Urug Cardiol. 2015;30 (3):295-311. 
16. O’Mahony C, Jichi F, Pavlou M, Monserrat L, Anastasakis A, Rapezzi C, et al. A novel clinical risk prediction model for sudden cardiac death in hypertrophic cardiomyopathy (HCM Risk-SCD). Eur Heart J. 2014;35(30):2010-20. doi: 10.1093/eurheartj/ peht439

17. Spirito $\mathbf{P}$, Autore $\mathbf{C}$, Rapezzi C, Bernabò P, Badagliacca R, Maron MS, et al. Syncope and risk of sudden death in hypertrophic cardiomyopathy. Circulation 2009;119(13):1703-10. doi: 10.1161/CIRCULATIONAHA.108.798314

18. Monserrat L, Elliott PM, Gimeno JR, Sharma S, Penas-Lado M, McKenna WJ. Nonsustained ventricular tachycardia in hypertrophic cardiomyopathy: an independent marker of sudden death risk in young patients. J Am Coll Cardiol. 2003;42 (5): 873-9.

19. Gimeno JR, Tome-Esteban M, Lofiego C, Hurtado J, Pantazis A, Mist B, et al. Exercise-induced ventricular arrhythmias and risk of sudden cardiac death in patients with hypertrophic cardiomyopathy. Eur Heart J. 2009;30(21):2599-605. doi: 10.1093/eurheartj/ehp327

20. Counihan PJ, Frenneaux MP, Webb DJ, McKenna WJ. Abnormal vascular responses to supine exercise in hypertrophic cardiomyopathy. Circulation 1991;84 (2):686-96.

21. Frenneaux MP, Counihan PJ, Caforio AL, Chikamori T, McKenna WJ. Abnormal blood pressure response during exercise in hypertrophic cardiomyopathy. Circulation 1990;82 (6):1995-2002.

22. Sadoul N, Prasad K, Elliott PM, Bannerjee S, Frenneau MP, McKenna WJ. Prospective prognostic assessment of blood pressure response during exercise in patients with hypertrophic cardiomyopathy. Circulation 1997;96 (9):2987-91.

23. Elliott PM, Gimeno JR, Tomé MT, Shah J, Ward D, Thaman R, et al. Left ventricular outflow tract obstruction and sudden death risk in patients with hypertrophic cardiomyopathy. Eur Heart J. 2006;27 (16):1933-41.

24. Maron MS, Olivotto I, Betocchi S, Casey SA, Lesser JR, Losi MA, et al. Effect of left ventricular outflow tract obstruction on clinical outcome in hypertrophic cardiomyopathy. N Engl J Med. 2003;348 (4):295-303. doi:10.1056/NEJMoa021332

25. Elliott P, Gimeno-Blanes JR, Mahon NG, Poloniecki J, McKenna WJ. Relation between severity of left-ventricular hypertrophy and prognosis in patients with hypertrophic cardiomyopathy. Lancet 2001; 357 (9254): 420-4. doi:10.1016/S0140-6736 (00)04005-8

26. Spirito $\mathbf{P}$, Bellone $\mathbf{P}$, Harris $\mathbf{K M}$, Benabo $\mathbf{P}$, Bruzzi P, Maron BJ. Magnitude of left ventricular hypertrophy and risk of sudden death in hypertrop- hic cardiomyopathy. N Engl J Med. 2000; 342 (24): 1778-85. doi: 10.1056/NEJM200006153422403

27. Maron MS. The role of cardiovascular magnetic resonance in sudden death risk stratification in hypertrophic cardiomyopathy. Card Electrophysiol Clin. 2015;7 (2):187-193. doi: 10.1016/j.ccep.2015.03.003

28. O'Hanlon R, Grasso A, Roughton M, Moon JC, Clark S, Wage R, et al. Prognostic significance of myocardial fibrosis in hypertrophic cardiomyopathy. J Am Coll Cardiol. 2010;56 (11):867-874. doi: 10.1016/j.jacc.2010.05.010

29. Chan RH, Maron BJ, Olivotto I, Pencina MJ, Assenza GE, Haas T, et al. Prognostic value of quantitative contrast enhanced cardiovascular magnetic resonance for the evaluation of sudden death risk in patients with hypertrophic cardiomyopathy. Circulation. 2014;130 (6):484-95. doi: 10.1161/ CIRCULATION AHA.113.007094.

30. McKenna WJ, Nagueh SF. Cardiac magnetic resonance imaging and sudden death risk in patients with hypertrophic cardiomyopathy. Circulation. 2014;130 (6):455-457. doi: 10.1161/CIRCULATIONAHA.114. 010977

31. Maron BJ. Clinical course and management of hypertrophic cardiomyopathy. N Engl J Med 2018; 379 (7): 655-68. doi: 10.1056/NEJMra1710575

32. Todiere G, Aquaro GD, Piaggi P, Formisano F, Barison A, Masci PG, et al. Progression of myocardial fibrosis assessed with cardiac magnetic resonance in hypertrophic cardiomyopathy. J Am Coll Cardiol. 2012;60 (10):922-9. doi: 10.1016/j.jacc.2012. 03.076

33. Moss AJ, ZarebaW, Hall WJ, Klein H, Wilber DJ, Cannom DS, et al. Prophylactic implantation of a defibrillator in patients with myocardial infarction and reduced ejection fraction. N Engl J Med 2002;346 (12):877-83. doi:10.1056/NEJMoa013474

34. Bardy GH, Lee KL, Mark DB, Poole JE, Packer DL, Boineau R, et al. Amiodarone or an implantable cardioverter-defibrillator for congestive heart failure. N Engl J Med. 2005;352 (3):225-37. Fe de errata en: N Engl J Med. 2005 May 19;352(20):2146. doi: 10.1056/NEJMoa043399

35. Ruiz-Salas A, García-Pinilla M, Cabrera-Bueno F, Fernández-Pastor J, Peña-Hernández J, Medina-Palomo C, et al. Comparison of the new risk prediction model (HCMRisk-SCD) and classic risk factors for suddendeath in patients with hypertrophic cardiomyopathy and defibrillator. Europace 2016;18 (5):773-7. doi: 10.1093/europace/euv079

36. Vriesendorp PA, Schinkel AFL, Liebregts M, Theuns D, Cleemput J, Cate FJ, et al. Validation of the 2014 European Society of Cardiology guidelines risk prediction model for the primary prevention of sudden cardiac death in hypertrophic cardiomyo- 
pathy. Circ Arrhythm Electrophysiol. 2015;8 (4):829-835. doi: 10.1161/CIRCEP.114.002553

37. Pedemonte O, Ferrando-Castagnetto F, Torterolo A, Ricca-Mallada R, Marichal P. Estratificación del riesgo de muerte súbita en un caso de miocardiopatía hipertrófica con fragmentación del complejo QRS. Rev Urug Cardiol 2016; 31: 61-6.

38. Maron BJ. Sudden death in young athletes. N Engl J Med. 2003;349 (11):1064-75. doi: 10.1056/ NEJMra022783

39. Maron BJ, Doerer JJ, Haas TS, Tierney DM, Mueller FO. Sudden deaths in young competitive athletes: analysis of 1866 deaths in the United States, 1980-2006. Circulation. 2009;119 (8):1085-92. doi: 10.1161/CIRCULATIONAHA.108.804617

40. Maron BJ, Udelson JE, Bonow RO, Nishimura RA, Ackerman MJ, Estes MA, et al. Eligibility and disqualification recommendations gor competitive athletes with cardiovascular anormalities: Task force 3: Hypertrophic cardiomyopathy, arrhytmogenicrigth ventricular cardiomyopathy ando other cardiomyopathies and miocarditis. A scientific statement from the American Heart Association and American College of Cardiology. Circulation 2015;132 (22):e273-80. doi: 10.1161/ CIR. 0000000000000239

41. Kiernan TJ, Weivoda PL, Somers VK, Ommen SR, Gersh BJ. Circadian rhythm of appropriate implantable cardioverter defibrillator discharges in patients with hypertrophic cardiomyopathy. Pacing Clin Electrophysiol. 2008;31 (10):1253-58. doi: 10.1111/j 1540- 8159.2008.01174.x

42. McKenna WJ, Oakley CM, Krikler DM, Goodwin JF. Improved survival with amiodarone in pa- tients with hypertrophic cardiomyopathy and ventricular tachycardia. Br Heart J. 1985;53 (4):412-6.

43. Melacini P, Maron BJ, Bobbo F, Basso C, Tokajuk B, Zucchetto M, et al. Evidence that pharmacological strategies lack efficacy for the prevention of sudden death in hypertrophic cardiomyopathy. Heart 2007;93 (6):708-10. doi:10.1136/hrt.2006. 099416

44. Begley DA, Mohiddin SA, Tripodi D, Winkler JB, Fananapazir L. Efficacy of implantable cardioverter defibrillator therapy for primary and secondary prevention of sudden cardiac death in hypertrophic cardiomyopathy. Pacing Clin Electrophysiol. 2003;26 (9):1887-96

45. Maron BJ, Spirito P, Shen WK, Haas TS, Formisano F, Link MS, et al. Implantable cardioverter-defibrillators and prevention of sudden cardiac death in hypertrophic cardiomyopathy. JAMA 2007;298 (4):405-12. doi:10.1001/jama.298.4.405

46. O'Mahony C, Lambiase PD, Quarta G, Cardona $\mathbf{M}$, Calcagnino $\mathbf{M}$, Tsovolas $\mathbf{K}$, et al. The long-term survival and the risks and benefits of implantable cardioverter defibrillators in patients with hypertrophic cardiomyopathy. Heart 2012;98 (2):116-125. doi: 10.1136/hrt.2010.217182

47. Bilge AK, Ozben B, Demircan S, Cinar M, Yilmaz E, Adalet K. Depression and anxiety status of patients with implantable cardioverter defibrillator and precipitating factors. Pacing Clin Electrophysiol. 2006;29 (6):619-26. doi:10.1111/j.1540-8159. 2006.00409.x

48. Pasipoularides A. Retos y controversias en miocardiopatía hipertrófica: visión integral desde la investigación básica, clínica y genética. Rev Esp Cardiol. 2018;71 (3):132-8 doi: 10.1016/j.recesp.2017.06.034 